\title{
Impact of blood count recovery on outcomes of acute myeloid leukemia patients achieving morphologic leukemia- free state
}

\author{
Wen-Yan Cheng ${ }^{1}$, Yong-Mei Zhu' ${ }^{1}$ Zhao Liu' ${ }^{1}$ Xiang-Qin Weng ${ }^{1}$, Jing-Ni Sui , Yun-Shuo Chen ${ }^{1}$, Shi-Yang Wang ${ }^{1}$ and \\ Yang Shen ${ }^{1}$
}

Acute myeloid leukemia (AML) is a group of heterogeneous hematological malignancies. Clinical features such as age, performance status, and cytogenetic and molecular abnormalities at diagnosis are all pre-treatment prognostic factors. In recent years, post-treatment parameters including minimal residual disease (MRD) and blood count recovery at the time of attaining morphologic leukemia-free state (MLFS) have drawn much research interest and considered to be significant in predicting the risk of relapse and survival. The concept of complete remission with incomplete hematologic recovery (CRi), fulfilling all $\mathrm{CR}$ criteria except for absolute neutrophil count $<1000$ per $\mu \mathrm{L}$ or platelet count $<100,000$ per $\mu \mathrm{L}$ in peripheral blood, was first proposed by Cheson et al. ${ }^{1}$, who suggested that patients with CRi had a relatively poor outcome compared with $\mathrm{CR}$ especially during initial chemotherapy. Subsequently, Larson et al. ${ }^{2}$ reported a significantly better relapse-free survival (RFS) rate in patients with $\mathrm{CR}$ than $\mathrm{CR}$ with incomplete platelet recovery (CRp, CR except for platelet count $<100,000$ per $\mu \mathrm{L})$ who did not receive further treatment beyond gemtuzumab ozogamicin. In a large series ${ }^{3}$, achievement of CRp rather than CR was independently associated with an inferior RFS. The long-term survival of CRi and its correlation with other clinical parameters, especially cytogenetic and molecular abnormalities and MRD, remain to be explored. Here we report a study of patients with de

\footnotetext{
Correspondence: Yang Shen (shen_yang@126.com)

'Department of Hematology, Shanghai Institute of Hematology, Ruijin Hospital Affiliated to Shanghai Jiao Tong University School of Medicine, Shanghai, China

These authors contributed equally: Wen-Yan Cheng, Yong-Mei Zhu, Zhao Liu.
}

novo AML who achieved CR or CRi after the first two cycles of chemotherapy and make comparisons between the two groups.

From January 2014 through December 2016, 350 consecutive newly diagnosed non-M3 adult AML patients were enrolled in this study. Patients with AML secondary to antecedent hematologic disorders were excluded. Cytogenetic risk categories were defined according to NCCN guidelines ${ }^{4}$. Younger patients $($ age $\leq 60)$ and fit elderly patients received standard first-line " $3+7$ " induction regimens, and high-dose cytarabine-based consolidation therapy. Unfit patients underwent either low-dose first-line regimens or other low-intensity therapy. Patients who met the standards of MLFS but failed to acquire neutrophil or platelet recovery after induction or first cycle of consolidation therapy were classified into CRi group. This study was approved by the ethic committee of Ruijin hospital. All patients had given informed consent according to the Declaration of Helsinki.

Gene mutations/fusions including FLT3-ITD and -TKD, C-KIT, N-RAS, CEBPA, DNMT3A, NPM1, MLL, IDH2, AML1-ETO, and CBFß-MYH11 were detected as previously reported ${ }^{5}$. Detection of MRD was based on leukemia-associated immunophenotype (LAIP) at diagnosis and performed by using 10-color multiparametric flow cytometry.

Kaplan-Meier method was used to calculate the distribution of overall survival (OS) and RFS. Cox proportional hazards model was applied for multivariate analysis of OS and RFS, and variables with $P$ value below 0.20 in univariate analysis were chosen to form the final model. 

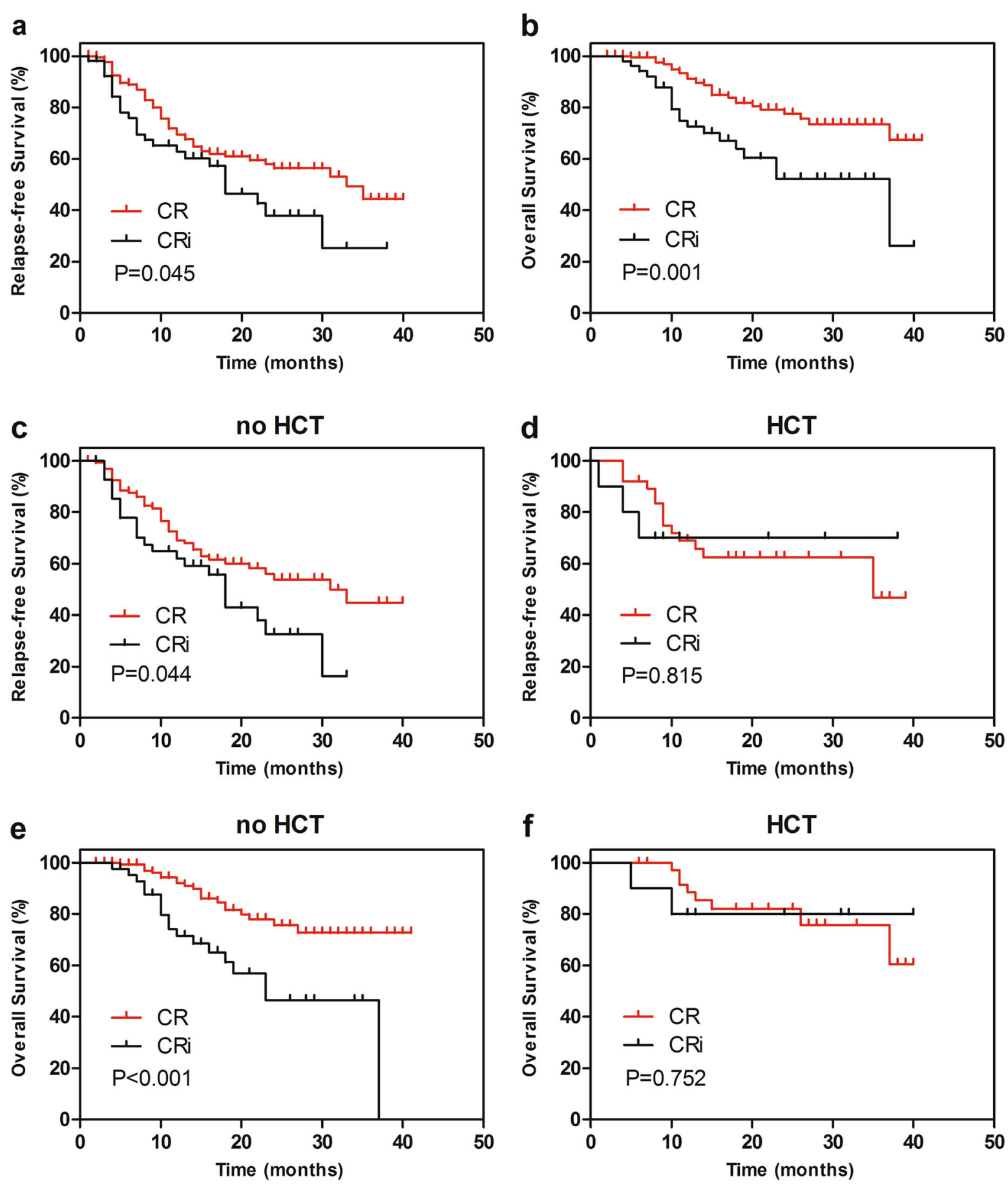

Fig. 1 Kaplan-Meier curves for probability of relapse-free and overall survival. a Relapse-free survival (RFS) for patients who achieved complete remission (CR) or CR with incomplete hematologic recovery (CRi). b Overall survival (OS) for patients who achieved CR or CRi. c, e RFS and OS for patients with CR or CRi who did not receive HCT. d, f RFS and OS for patients with CR or CRi who received HCT

Clinical characteristics of the 350 patients are summarized (Supplementary Table 1). Among which, 26 patients died early, and 14 patients lost contact after induction therapy. Among the remaining 310 patients whose responses were evaluable, 230 patients $(74.2 \%)$ achieved either CR $(n=179)$ or CRi $(n=51), 57$ patients (18.4\%) and 23 patients (7.4\%) showed no remission (NR) and partial remission (PR), respectively. Further analyses 
Table 1 Univariate and multivariate analyses for relapse-free and overall survival

\begin{tabular}{|c|c|c|c|c|c|c|c|c|}
\hline \multirow[t]{3}{*}{ Factor } & \multicolumn{4}{|l|}{ RFS } & \multicolumn{4}{|l|}{ os } \\
\hline & \multicolumn{2}{|l|}{ Univariate } & \multicolumn{2}{|l|}{ Multivariate } & \multicolumn{2}{|l|}{ Univariate } & \multicolumn{2}{|l|}{ Multivariate } \\
\hline & HR (95\% Cl) & $P$ value & HR (95\% Cl) & $P$ value & HR $(95 \% \mathrm{Cl})$ & $P$ value & HR $(95 \% \mathrm{Cl})$ & $P$ value \\
\hline Age & $1.026(1.012-1.041)$ & $<0.001$ & $1.022(1.005-1.039)$ & 0.010 & $1.044(1.024-1.065)$ & $<0.001$ & $1.051(1.025-1.077)$ & $<0.001$ \\
\hline WBC $\left(\times 10^{9} / L,>30\right.$ vs. $\left.\leq 30\right)$ & $1.583(1.025-2.445)$ & 0.038 & & NS & $1.070(0.581-1.970)$ & 0.829 & & \\
\hline \multicolumn{9}{|l|}{ Cytogenetics } \\
\hline Intermediate vs. favorable & $0.754(0.430-1.322)$ & 0.324 & & & $0.890(0.421-1.881)$ & 0.760 & & \\
\hline Unfavorable vs. favorable & $1.241(0.530-2.906)$ & 0.620 & & & $1.881(0.667-5.303)$ & 0.232 & & \\
\hline CRi vs. CR & $1.582(0.999-2.507)$ & 0.051 & $1.869(1.087-3.213)$ & 0.024 & $2.546(1.439-4.505)$ & 0.001 & $3.257(1.624-6.530)$ & 0.001 \\
\hline MRD (positive vs. negative) & $1.411(0.866-2.297)$ & 0.167 & & NS & $1.970(1.032-3.761)$ & 0.040 & & NS \\
\hline FLT3-ITD & $1.773(1.000-3.143)$ & 0.050 & & NS & $1.300(0.581-2.911)$ & 0.523 & & \\
\hline$N P M 1 \mathrm{~m}^{+} / F L T 3-\mathrm{ITDm}^{-}$ & $0.834(0.471-1.478)$ & 0.534 & & & $1.015(0.492-2.092)$ & 0.968 & & \\
\hline MLL-fusion gene & $3.083(1.223-7.705)$ & 0.016 & $3.514(1.045-11.815)$ & 0.042 & $1.863(0.447-7.760)$ & 0.393 & & \\
\hline MLL-PTD & $2.456(0.990-6.096)$ & 0.053 & $2.937(1.039-8.303)$ & 0.042 & $3.623(1.290-10.174)$ & 0.015 & $5.360(1.569-18.319)$ & 0.007 \\
\hline$C-K I T$ & $1.508(0.849-2.678)$ & 0.161 & $2.555(1.365-4.782)$ & 0.003 & $1.670(0.807-3.454)$ & 0.167 & $3.670(1.626-8.283)$ & 0.002 \\
\hline Biallelic CEBPA & $0.636(0.364-1.109)$ & 0.111 & & NS & $0.465(0.198-1.092)$ & 0.079 & & NS \\
\hline $\mathrm{HCT}$ & $0.803(0.472-1.366)$ & 0.419 & & & $0.761(0.379-1.530)$ & 0.444 & & \\
\hline
\end{tabular}

RFS relapse-free survival, $O S$ overall survival, $H R$ hazard ratio, $C l$ confidence interval, $W B C$ white blood cell, $C R$ complete remission, $C R i$ complete remission with incomplete hematologic recovery, MRD minimal residual disease, HCT hematopoietic cell transplantation

were mainy conducted in the $\mathrm{CR}$ and CRi cohorts. At diagnosis, age, white blood count, hemoglobin level, and bone marrow (BM) blasts were similar between the two groups. Principal difference was the lower platelet count in CRi patients than those with CR (median, $29 \times 10^{9} / \mathrm{L}$ vs. $\left.49 \times 10^{9} / \mathrm{L}, P=0.024\right)$. Patients achieving CRi were prone to having less favorable cytogenetics ( $8 \%$ vs. $19.4 \%$, $P=0.06$ ), which was approximate to those with PR or NR (8.2\%). Forty-eight patients (20.9\%) with CR or CRi underwent hematopoietic cell transplantation (HCT) at least after two courses of treatment.

In comparison to patients with $\mathrm{CR}$, patients in $\mathrm{CRi}$ group had a lower incidence of C-KIT mutations ( $4 \%$ vs. $14.9 \%, P=0.039)$ and a higher frequency of biallelic CEBPA mutations (biCEBPA, $33.3 \%$ vs. $19.6 \%, P=0.038$ ). We then classified gene abnormalities into three subgroups per 2017 ELN recommendations ${ }^{6}$, and found that the percentages of favorable, intermediate, and adverse genetic alterations were similar among $\mathrm{CR}$ and CRi group (Supplementary Table 2).

A total of 196 patients (85.2\%) with CR or CRi presented definite LAIP and had MRD results after induction therapy. Patients with CRi had a significantly higher MRD level compared with those with CR (median, $0.046 \%$ vs. $0.017 \% ; P=0.007$ ). Similarly, a higher frequency of positive MRD (MRD $\geq 0.1 \%$ ) could be seen in patients with CRi (45.2\% vs. $22.7 \%, P=0.004$ ) (Supplementary Table 3).

Patients in CR group had a longer RFS compared with those whose best response was CRi $(P=0.045$, Fig. 1a), with an estimated median RFS of 33 and 18 months, respectively. Similarly, patients with $\mathrm{CR}$ had a significantly better OS than CRi $(P=0.001$, Fig. $1 b)$. The estimated median OS was 37 months in patients with CRi while it was "not reached" in those with CR. As presented in Fig. $1 \mathrm{c}-\mathrm{f}$, for patients who did not receive HCT, failure in achieving blood count recovery conferred a shorter RFS (median RFS, 31 months for CR, 18 months for CRi; $P=$ 0.044) and OS (median OS, "not reached" for CR, 23 months for CRi; $P<0.001)$. While for those who underwent $\mathrm{HCT}$, including 9 patients $(17.6 \%)$ with CRi and 39 patients $(21.8 \%$ ) with $\mathrm{CR}$, the negative effect of CRi on both RFS (median RFS, 35 months for CR, "not reached" for CRi; $P=0.815$ ) and OS (median OS, "not reached" for both groups; $P=0.752$ ) was greatly attenuated.

We conducted univariate and multivariate analyses for RFS and OS (Table 1). After accounting for covariates, achievement of CRi other than CR was an independent factor associated with shorter RFS (hazard ratio (HR), 1.869; 95\% confidence interval (CI), 1.087-3.213; $P=$ $0.024)$ and $\mathrm{OS}(\mathrm{HR}, 3.257 ; 95 \% \mathrm{CI}, 1.624-6.530 ; P=$ 
0.001). Age, $M L L$-fusion genes, $M L L$-PTD, and C-KIT mutations were also significant factors related to prognosis.

Prior reported data demonstrated CRi or CRp as an independent factor related to shorter duration of RFS, but less relavant to $\mathrm{OS}^{2,3}$. In other cases, however, CRi lost prognostic value in multivariate analysis ${ }^{7,8}$. Our study indicated that even when other recognized prognostic factors were considered, CRi was independently associated with inferior RFS and OS. Two aspects may underlie the relatively poor outcomes of CRi. Firstly, the frequency of favorable cytogenetics was lower in patients with $\mathrm{CRi}$ than those with $\mathrm{CR}$, which may reflect the importance of cytogenetic risk in determining the depth of remission. Moreover, MRD level after therapy is a wellrecognized prognostic factor in both acute lymphoblastic leukemia and $\mathrm{AML}^{8,9}$. We observed that patients with $\mathrm{CRi}$ were more likely to have a higher MRD level and greater frequency of MRD positive status than those with CR after initial induction, which was also observed by Chen et al. ${ }^{10}$ who revealed a strong correlation between MRD and response. We speculate that level of blood count at remission may reflect the amount of minimal residual leukemic cells, which may disrupt hematopoietic regeneration by their toxicity to normal progenitor cells. However, the unfavorable prognostic effect of CRi was greatly attenuated among patients whose MRD was eliminated through $\mathrm{HCT}$, which was similar to the results of the study by Chen et al. ${ }^{10}$ These findings suggest that in combination of blood count and MRD at remission, we are probable to identify patients at a higher risk of relapse and prevent it by using more intensive chemotherapy or selecting novel therapeutic agents to elimilate residual leukemia cells and following with HCT, which may improve the outcome of patients with CRi and could be a direction of future clinical trials.

We previously reported that CEBPA mutations accounted for $22 \%$ of cytogenetically normal AML (CN$\mathrm{AML})$ in a large series ${ }^{5}$, a propotion that was higher compared with $12.8 \%$ in a $1182 \mathrm{CN}$-AML cohort $^{11}$, which may reflect the difference in genetic backgrounds between Chinese patients and their European counterparts. However, the long-term prognosis of biCEBPA in our cohort (3-year OS rate, $57 \%)^{5}$ was not as good as the results of the study by Taskesen et al. ${ }^{11}$ (5-year OS rate, $63 \%$ ), which might be partially because that patients with $\operatorname{biCEBPA}$ in our cohort were more likely to achieve CRi. Notably, patients with biCEBPA in this study presented a lower platelet count at diagnosis compared with those without (median, $24 \times 10^{9} / \mathrm{L}$ vs. $49 \times 10^{9} / \mathrm{L}, \quad P<0.001$; Supplementary Table 4 ), which was also observed by Taskesen et al. ${ }^{11}$ These results suggest that CEBPA mutations may exert adverse impact on hematopoietic differentiation and recovery of blood count after chemotherapy. In contrast, although we $\mathrm{w}^{12}$ and others ${ }^{13}$ have reported that C-KIT mutations were adverse events in core binding factor leukemias, they were less common in CRi patients compared with those who achieved CR, which needs to be verified in large population.

In summary, our study demonstrates that achieving CRi rather than CR after early cycles of chemotherapy independently predicts shorter RFS and OS. Monitoring the quality of remission through blood count is a straightforward and convenient way to assess the risk of relapse and survival, which should be considered in therapeutic decision-making after remission.

\section{Acknowledgements}

This work was supported in part by the National Natural Science Foundation of China (no. 81770141), the National Key R\&D Program of China (no.

2016YFE0202800), and Shanghai Municipal Education Commission-Gaofeng Clinical Medicine Grant Support (no. 20161406).

Conflict of interest

The authors declare that they have no conflict of interest.

\section{Publisher's note}

Springer Nature remains neutral with regard to jurisdictional claims in published maps and institutional affiliations.

Supplementary Information accompanies this paper at https://doi.org/ 10.1038/s41408-018-0094-3.

Received: 29 January 2018 Revised: 27 March 2018 Accepted: 9 April 2018 Published online: 07 June 2018

\section{References}

1. Cheson, B. D. et al. Revised recommendations of the International Working Group for Diagnosis, Standardization of Response Criteria, Treatment Outcomes, and Reporting Standards for Therapeutic Trials in Acute Myeloid Leukemia. J. Clin. Oncol. 21, 4642-4649 (2003).

2. Larson, R. A. et al. Final report of the efficacy and safety of gemtuzumab ozogamicin (Mylotarg) in patients with CD33-positive acute myeloid leukemia in first recurrence. Cancer 104, 1442-1452 (2005).

3. Walter, R. B. et al. Effect of complete remission and responses less than complete remission on survival in acute myeloid leukemia: a combined Eastern Cooperative Oncology Group, Southwest Oncology Group, and M. D. Anderson Cancer Center Study. J. Clin. Oncol. 28, 1766-1771 (2010).

4. O'Donnell, M. R. et al. Acute myeloid leukemia, version 3.2017, NCCN Clinical Practice Guidelines in Oncology. J. Natl. Compr. Canc. Netw. 15, 926-957 (2017).

5. Shen, Y. et al. Gene mutation patterns and their prognostic impact in a cohort of 1185 patients with acute myeloid leukemia. Blood 118, 5593-5603 (2011).

6. Dohner, H. et al. Diagnosis and management of AML in adults: 2017 ELN recommendations from an international expert panel. Blood 129, 424-447 (2017).

7. Araki, D. et al. Allogeneic hematopoietic cell transplantation for acute myeloid leukemia: time to move toward a minimal residual disease-based definition of complete remission? J. Clin. Oncol. 34, 329-336 (2016).

8. Ravandi, F. et al. Persistence of minimal residual disease assessed by multiparameter flow cytometry is highly prognostic in younger patients with acute myeloid leukemia. Cancer 123, 426-435 (2017).

9. Weng, X. Q. et al. Prognostic significance of monitoring leukemia-associated immunophenotypes by eight-color flow cytometry in adult B-acute lymphoblastic leukemia. Blood Cancer J. 3, e133 (2013). 
10. Chen, $X$. et al. Relation of clinical response and minimal residual disease and their prognostic impact on outcome in acute myeloid leukemia. J. Clin. Oncol. 33, 1258-1264 (2015).

11. Taskesen, E. et al. Prognostic impact, concurrent genetic mutations, and gene expression features of AML with CEBPA mutations in a cohort of 1182 cytogenetically normal AML patients: further evidence for CEBPA double mutant AML as a distinctive disease entity. Blood 117, 2469-2475 (2011).
12. Chen, Y. S. et al. Kinase-associated gene mutation pattern and clinical relevance in 205 patients with core binding factor leukemias. Blood Cancer J. 6, e494 (2016).

13. Qin, Y. Z. et al. Prevalence and prognostic significance of c-KIT mutations in core binding factor acute myeloid leukemia: a comprehensive large-scale study from a single Chinese center. Leuk. Res. 38, 1435-1440 (2014). 